\title{
Identificación y estudio de susceptibilidad antimicrobiana de bacterias potencialmente responsables de infecciones nosocomiales en los hospitales veterinarios de la Universidad de Chile*
}

\author{
MARÍA ANTONIETA JARA O., M.V, M.C.V., PAULINA AVENDAÑO R., L.C.V. \\ y CARLOS NAVARRO V., B.Q., M.C.V.
}

Departamento de Medicina Preventiva Animal. Facultad de Ciencias Veterinarias y Pecuarias. Universidad de Chile.

\author{
ABSTRACT \\ IDENTIFICATION AND SUSCEPTIBILITY PROFILE OF POTENTIALLY \\ NOSOCOMIALS BACTERIA IN A VETERINARY TEACHING HOSPITAL FROM \\ UNIVERSITY OF CHILE
}

The majority of the nosocomials bacteria have demonstrated an increase in the resistance to antibiotic used habitually in the veterinary hospitals. Thus, in this work we isolate, identify and determine the susceptibility profile of 48 environmental nosocomial bacteria strains from the clinical veterinary hospitals of the University of Chile: Enterococcus faecium, Enterobacter cloacae, Escherichia coli, Pseudomonas aeruginosa, Staphylococcus kloossii, Staphylococcus intermedius, Pantoea agglomerans, Citrobacter freundii, Acinetobacter baumannii and Staphylococcus epidermidis. Due to $85 \%$ of the nosocomials strains showed the multiresistance phenomenon, the susceptibility profile represent a great impact and a serious problem in adequate therapy election. Thus, a worrying reality not only present in the analyzed hospitals is suggested.

Key words: Nosocomials infections, small animals.

\section{RESUMEN}

Debido a que la mayoría de la bacterias nosocomiales han demostrado un aumento de la resistencia a los antibióticos usados habitualmente en los hospitales veterinarios, en este trabajo se consideró realizar un estudio en los hospitales clínicos veterinarios de la Universidad de Chile con el objetivo de aislar, identificar y determinar el perfil de sensibilidad de cepas bacterianas ambientales potencialmente responsables de infecciones nosocomiales. Así, se logró aislar y caracterizar 48 cepas de bacterias descritas como nosocomiales según literatura: Enterococcus faecium, Enterobacter cloacae,

* Financiado por Proyecto FIV 4602016. Dirección de Investigación. Facultad de Ciencias Veterinarias y Pecuarias. Universidad de Chile.

E-mail: mjara@uchile.cl 
M. A. JARA O. y cols.

Escherichia coli, Pseudomonas aeruginosa, Staphylococcus kloossii, Staphylococcus intermedius, Pantoea agglomerans, Citrobacter freundii, Acinetobacter baumannii y Staphylococcus epidermidis. El perfil de sensibilidad obtenido tiene un gran impacto, ya que el $85 \%$ de las cepas nosocomiales analizadas mostró el fenómeno de multiresistencia. Lo anterior representa tanto un problema serio en la elección de terapia adecuada como también sugiere una realidad preocupante presente no sólo en los hospitales analizados.

Palabras clave: Infección nosocomial, pequeños animales.

\section{INTRODUCCIÓN}

Las infecciones nosocomiales son aquellas que no están presentes ni en periodo de incubación cuando el paciente ingresa al recinto hospitalario, y en general se considera que éstas se presentan posterior a las 48-72 horas del ingreso al centro asistencial (Organización Mundial de la Salud, (OMS) 2003). Estas infecciones tienen diferentes agentes etiológicos, siendo los más comunes bacterias, virus, parásitos y hongos. En el caso de las bacterias, se ha descrito que estos patógenos pueden ser adquiridos por los pacientes a través de un mecanismo de transmisión cruza$\mathrm{da}$, es decir, desde otros animales hospitalizados que constantemente liberan microorganismos al medio ambiente. Por otra parte, otra forma de transmisión es a través de los objetos inanimados como estetoscopios, sondas de ultrasonido, catéteres urinarios e intravenosos; sin embargo, la principal fuente de infección nosocomial corresponde a las manos del personal médico, lo cual probablemente se debe a la inadecuada desinfección de sus manos entre la manipulación de los distintos pacientes (OMS, 2003).

Con respecto a los factores de riesgo, los manejos y procedimientos realizados en cuidados intensivos, el uso de aparatos y procedimientos invasivos y la hospitalización prolongada, aumentan el riesgo de contraer una infección nosocomial (Johnson, 2002). Este tema adquiere real importancia considerando que en la actualidad, la medicina veterinaria se asemeja enormemente a la medicina humana en lo que respecta a los distintos manejos y técnicas exploratorias, implementándose cada vez más, manejos de cuidados intensivos en nuestros animales. Por otro lado, es necesario insistir que la prevención es el factor más importante para el control de estas infecciones y de ahí la recomendación de implementar un protocolo de limpieza, considerando la frecuencia de aseo, tipos de desinfectantes, práctica de lavado de manos, además del uso de técnicas asépticas en el manejo de dispositivos invasivos, barreras de protección entre animales, disminución de los tiempos de hospitalización y determinación de los posibles vectores de microorganismos (Johnson, 2002).

En lo que respecta a los agentes bacterianos, estudios realizados en medicina humana demuestran que las bacterias causantes de infecciones nosocomiales han sufrido grandes cambios a lo largo del tiempo, y en un inicio los patógenos predominantes detectados fueron principalmente Gram positivos. Luego, con la introducción de los antibióticos se produjo una disminución de estas infecciones pasando a ser producidas mayoritariamente por bacterias Gram negativas (Alpuche y Daza, 2002). Entre los principales agentes bacterianos Gram positivos aislados en medicina humana, podemos mencionar las cocáceas Staphylococcus aureus meticilino-resistentes (Sasaki y col., 2007), y Enterococcus spp (DeLisle y Perl, 2003), principalmente E. faecium E. faecalis. Entre los bacilos Gram negativos no fermentadores Acinetobacter baumannii (Héritier y col., 2005) y Pseudomonas aeruginosa (Tsakris y col., 2000). Las enterobacterias Klebsiella pneumoniae (Sánchez y col., 2006) y Escherichia coli (Bean y col., 2008) como bacilos fermentadores.

En el caso de la medicina veterinaria, las bacterias descritas como nosocomiales no difieren mucho de las aisladas en humanos y así encontramos a Staphylococcus aureus meticilino-resistente (Cuny y col., 2006; Leonard y Markey, 2008) Acinetobacter baumannii (Francey y col., 2000), Pseudomonas aeruginosa, Enterococcus faecalis y Enterococcus faecium (Boerlin y col., 2001). La importancia de estas bacterias radica en que la gran mayoría de ellas son naturalmente 
resistentes a antimicrobianos o poseen la habilidad de adquirir esta resistencia. Así, estudios en medicina veterinaria han determinado la existencia de bacterias nosocomiales multirresistentes tales como Acinetobacter baumanni, Enterobacter spp. y Klebsiella spp. a lo largo de los años. Además, estos estudios revelan un aumento en la proporción de aislamientos de Enterococcus spp. lo cual podría ser indicativo de un incremento en la resistencia a antimicrobianos por parte de esta bacteria. En cuanto a Staphylococcus aureus, se ha determinado un cambio en la presentación de resistencia, aumentando para algunos antimicrobianos y disminuyendo para otros. (Prescott $y$ col., 2002).

La resistencia bacteriana representa la capacidad de los microorganismos de resistir la acción de los antimicrobianos. Actualmente, una bacteria se considera resistente cuando las concentraciones de un antimicrobiano necesarias para inhibir el crecimiento de ella in vitro, son mayores que las concentraciones alcanzadas en suero o en tejidos, medidos por la concentración inhibitoria mínima (MIC). La información que permite que una bacteria desarrolle un mecanismo de resistencia se encuentra en su material genético y por esta razón, la aparición y diseminación de mecanismos de resistencia se ve favorecida por la posibilidad que poseen las bacterias de modificar su material genético o de recibir material genético de forma horizontal por parte de otras bacterias (Alpuche y Daza, 2002).

De acuerdo a lo anterior, las bacterias pueden volverse resistentes como resultado de mutaciones de los genes cromosomales o bien, por intercambio de material genético mediante el transporte de genes de resistencia a través de mecanismos como transformación, transducción y conjugación, siendo este último el más importante (Jhonson, 2002).

El incremento de la resistencia bacteriana se ha visto favorecido por una serie de factores relacionados con el manejo inadecuado y el uso indiscriminado de antibióticos. Para evitar este problema se deben tomar ciertas medidas preventivas como limitar el uso profiláctico de antimicrobianos, con el fin de no ejercer una presión de selección que pueda inducir la aparición y diseminación de esta resistencia.

En la actualidad, el tópico infección nosocomial es un tema que ha sido intensamente estudiado en medicina humana por el significativo impacto que tiene en la salud publica, ocurriendo lo contrario en medicina veterinaria en donde existe poca información; sin embargo, lo publicado demuestra algunas tendencias similares a las encontradas en la medicina humana lo cual permite predecir un futuro semejante en nuestra profesión.

Considerando que la gran mayoría de las bacterias nosocomiales han demostrado un incremento en la resistencia a los antibióticos usados habitualmente en los hospitales veterinarios, se realizó un estudio en los hospitales clínicos veterinarios de la Universidad de Chile con el objetivo de aislar, identificar y determinar la susceptibilidad antimicrobiana de cepas bacterianas ambientales potencialmente responsables de infecciones nosocomiales.

\section{MATERIAL Y MÉTODOS}

\section{Diseño Experimental}

La realización de este trabajo contempló la búsqueda activa de bacterias ambientales descritas como nosocomiales según literatura y la determinación de su perfil de susceptibilidad antimicrobiana. Para esto, se tomaron muestras desde los hospitales clínicos veterinarios de la Universidad de Chile para el aislamiento e identificación bacteriana. Lo anterior en conjunto con la determinación de susceptibilidad antimicrobiana se realizó en la Unidad de Microbiología del Departamento de Medicina Preventiva Animal de la Facultad de Ciencias Veterinarias y Pecuarias de la Universidad de Chile, en el período 2007-2008.

\section{Muestras}

Para la realización de este estudio se recolectaron 380 muestras desde las diferentes dependencias que conforman los hospitales clínicos veterinarios de la Universidad de Chile, sedes Bilbao y Facultad, abarcando salas de hospitalización, consultas, pabellones quirúrgicos y salas de rayos X. En estos lugares se seleccionaron diferentes superficies a muestrear: jaulas, mesones de trabajo, muebles, máquinas de anestesia y de toma de radiografías. La recolección de muestras se realizó mediante tórulas estériles humedecidas en $0,5 \mathrm{ml}$ de suero fisiológico las cuales se deslizaron sobre las distintas superficies a muestrear $(5,0 \times 5,0 \mathrm{~cm})$ y luego depositadas en tubos 
venoject para ser, inmediatamente, transportadas al laboratorio para su procesamiento. En el laboratorio, cada tórula fue trasladada a un tubo de ensayo con $5 \mathrm{ml}$ de caldo soya tripticasa. Posteriormente, los tubos fueron agitados durante 5 minutos en vortex para finalmente ser incubados a $37^{\circ} \mathrm{C}$ durante 24 horas (Lemmen y col., 2001).

\section{Aislamiento Bacteriano}

Después de 24 horas de incubación, en los medios de cultivo en donde se observó desarrollo bacteriano, indicado por la turbidez del medio, se procedió a realizar el aislamiento bacteriano en agar soya tripticasa sangre y agar MacConckey. Una vez sembradas, ambas placas fueron incubadas a $37^{\circ} \mathrm{C}$, esperando su desarrollo hasta 48 horas.

\section{Estudio de las características morfológicas y tintoriales de las colonias}

Se observó el aspecto de las colonias desarrolladas en los medios de cultivo, analizándose características que permitieron seleccionar colonias de interés. Así, en las placas de agar soya tripticasa sangre se estudió la forma, tamaño, color y hemólisis de las colonias; mientras que en las placas de agar MacConckey se observó la coloración de las colonias, que permite distinguir entre aquellas que hidrolizan lactosa y por ende, adquieren una coloración rojiza, y las que no lo hacen y permanecen incoloras. Con todas estas características se seleccionaron aquellas colonias sospechosas de ser nosocomiales, procediéndose a realizar un frotis de ellas para la tinción de Gram. Esta información fue requerida para la identificación bacteriana que se realizó posteriormente.

\section{Identificación de cepas bacterianas}

Para la identificación de las cepas, se utilizó un kit diagnóstico comercial. En el caso de las colonias Gram (-) el kit BBL cristal de identificación de patógenos entéricos/no fermentadores y paralelamente se realizó la prueba del indol, requerida por el kit. La identificación de las colonias Gram (+) se realizó con el kit diagnóstico BBL cristal para Gram (+). En ambos casos se procedió según las indicaciones del fabricante.

\section{Determinación de la susceptibilidad antimicrobiana}

Todas las cepas fueron sometidas al método por difusión en placa según Kirby Bauer, según las normas del "National Committe for Clinical Laboratory Standards" (NCCLS, 1997). Como cepas controles se utilizaron Staphylococcus aureus ATCC 25923, Enterococcus faecalis ATCC 29212, Escherichia coli ATCC 25922, y Pseudomonas aeruginosa ATCC 27853. Se utilizaron los siguientes discos de antimicrobianos: Quinolonas (Enrofloxacino (Enr), Ciprofloxacino (Cip)), Betalactámicos con y sin inhibidores de beta lactamasas (Amoxicilina/Acido Clavulánico (Amc), Sulperazon (Sul), Oxacilina (Ox) Ampicilina (A)), Tetraciclinas (Tetraciclina (T), Doxiciclina (D)), Glicopéptidos (Vancomicina (V)) y Sulfas (Sulfa/trimetropin (Stx).

Se clasificará como cepa multiresistente a aquella que presente resistencia a dos o más de los antimicrobianos utilizados.

Tabla 1. Número de muestras y su composición (\%) en la búsqueda activa de bacterias nosocomiales, según sede

\begin{tabular}{lrrrr}
\hline Condición & Sede Bilbao & \% & Sede Facultad & \% \\
\hline Total de muestras & 210 & 100 & 170 & 100 \\
Muestras sin desarrollo & 41 & 20 & 4 & 2 \\
Muestras con desarrollo & 169 & 80 & 166 & 98 \\
Muestras eliminadas & 76 & 36 & 92 & 54 \\
Muestras en cepario & 93 & 44 & 74 & 44 \\
Cepas sometidas a estudio & 41 & 20 & 20 & 12 \\
Cepas Gram (+) & 17 & 11 & 10 & 8 \\
Cepas Gram (-) & 20 & 16 & 10 & 4 \\
Cepas nosocomiales & 37 & 2 & 11 & 6 \\
Otras cepas & 4 & & 9 & 5 \\
\hline
\end{tabular}




\section{RESULTADOS Y DISCUSIÓN}

Como se desglosa en la Tabla 1 , se logró obtener un cepario de 167 cepas, de ellas sólo 48 resultaron ser cepas descritas como nosocomiales por la literatura consultada. En "otras cepas" se incluyen a aquellas que no están descritas como nosocomiales y las que no fueron posibles de identificar por el método utilizado. Las muestras señaladas como "eliminadas" incluyeron aquellas con desarrollo polimicrobiano o pertenecientes al género Bacillus, sin relevancia según literatura. La Tabla 2 sugiere una mayor existencia de bacterias nosocomiales en la sede Bilbao, lo cual podría deberse a que en esta sede la cantidad de pacientes hospitalizados siempre fue mayor. Las
Tabla 2. Frecuencia de aislamiento bacteriano, según sede estudiada

\begin{tabular}{lrcr}
\hline $\begin{array}{l}\text { EspecieSede } \\
\text { bacteriana }\end{array}$ & $\begin{array}{c}\text { Sede } \\
\text { Bilbao }\end{array}$ & Facultad & Total \\
\hline E. faecium & 14 & 6 & 20 \\
E. cloacae & 9 & 0 & 9 \\
E. coli & 4 & 1 & 5 \\
P. aeruginosa & 4 & 0 & 4 \\
S. kloossii & 2 & 1 & 3 \\
S. intermedius & 1 & 2 & 3 \\
P. agglomerans & 1 & 0 & 1 \\
C. freundii & 1 & 0 & 1 \\
A. baumannii & 1 & 0 & 1 \\
S. epidermidis & 0 & 1 & 1 \\
Total & 37 & 11 & 48 \\
\hline
\end{tabular}

Tabla 3. Sensibilidad antimicrobiana en aislados Gram (+). Sedes Bilbao y Facultad (oscuro)

\begin{tabular}{|c|c|c|c|c|c|c|c|c|c|c|c|c|}
\hline $\mathbf{N}^{0}$ & Aislado & Especie & Enr & $\mathbf{A}$ & Amc & Cip & $\mathbf{T}$ & Stx & Van & D & $\mathbf{O x}$ & Sul \\
\hline 1 & 337 & E. faecium & $\mathrm{S}$ & $\mathrm{R}$ & $\mathrm{R}$ & $\mathrm{S}$ & $\mathrm{S}$ & $\mathrm{S}$ & $\mathrm{S}$ & $\mathrm{S}$ & $\mathrm{R}$ & $\mathrm{R}$ \\
\hline 2 & 341 & E. faecium & SI & $\mathrm{R}$ & $\mathrm{R}$ & $\mathrm{S}$ & $\mathrm{R}$ & $\mathrm{R}$ & $\mathrm{S}$ & SI & $\mathrm{R}$ & $\mathrm{R}$ \\
\hline 3 & $356-\mathrm{m}$ & E. faecium & $\mathrm{S}$ & $\mathrm{R}$ & $\mathrm{R}$ & $\mathrm{S}$ & $\mathrm{R}$ & $\mathrm{S}$ & $\mathrm{S}$ & $\mathrm{R}$ & $\mathrm{R}$ & $\mathrm{R}$ \\
\hline 4 & $358-\mathrm{s}$ & E. faecium & SI & $\mathrm{R}$ & $\mathrm{R}$ & SI & $\mathrm{S}$ & $\mathrm{R}$ & SI & SI & $\mathrm{R}$ & $\mathrm{R}$ \\
\hline 5 & 001-3 & E. faecium & $\mathrm{R}$ & $\mathrm{R}$ & S I & S I & $\mathrm{R}$ & $\mathrm{S}$ & $\mathrm{S}$ & $\mathrm{R}$ & $\mathrm{R}$ & $\mathrm{R}$ \\
\hline 6 & 009-2 & E. faecium & $\mathrm{R}$ & $\mathrm{R}$ & S I & $\mathrm{R}$ & $\mathrm{S}$ & $\mathrm{S}$ & $\mathrm{S}$ & $\mathrm{S}$ & $\mathrm{R}$ & $\mathrm{R}$ \\
\hline 7 & 014-1 & E. faecium & $\mathrm{S}$ & $\mathrm{S}$ & S & S I & $\mathrm{R}$ & $\mathrm{S}$ & $\mathrm{S}$ & S I & $\mathrm{R}$ & S I \\
\hline 8 & 036-1 & E. faecium & S I & $\mathrm{R}$ & $\mathrm{R}$ & S I & $\mathrm{R}$ & $\mathrm{S}$ & $\mathrm{S}$ & $\mathrm{R}$ & $\mathrm{R}$ & $\mathrm{R}$ \\
\hline 9 & 038-2 & E. faecium & S I & $\mathrm{S}$ & $\mathrm{S}$ & S I & $\mathrm{R}$ & $\mathrm{S}$ & $\mathrm{S}$ & $\mathrm{R}$ & $\mathrm{R}$ & S I \\
\hline 10 & 061 & E. faecium & $\mathrm{R}$ & $\mathrm{R}$ & S I & $\mathrm{R}$ & $\mathrm{R}$ & $\mathrm{R}$ & $\mathrm{S}$ & $\mathrm{R}$ & $\mathrm{R}$ & $\mathrm{R}$ \\
\hline 11 & 051 & E. faecium & $\mathrm{R}$ & $\mathrm{R}$ & $\mathrm{R}$ & $\mathrm{R}$ & $\mathrm{R}$ & $\mathrm{R}$ & $\mathrm{S}$ & $\mathrm{R}$ & $\mathrm{R}$ & $\mathrm{R}$ \\
\hline 12 & 076 & E. faecium & $\mathrm{R}$ & $\mathrm{R}$ & $\mathrm{R}$ & $\mathrm{R}$ & $\mathrm{R}$ & $\mathrm{R}$ & $\mathrm{S}$ & $\mathrm{R}$ & $\mathrm{R}$ & $\mathrm{R}$ \\
\hline 13 & 082 & E. faecium & $\mathrm{R}$ & $\mathrm{R}$ & $\mathrm{R}$ & $\mathrm{R}$ & $\mathrm{R}$ & $\mathrm{R}$ & $\mathrm{S}$ & $\mathrm{R}$ & $\mathrm{R}$ & $\mathrm{R}$ \\
\hline 14 & 317 & E. faecium & $\mathrm{S}$ & $\mathrm{R}$ & SI & SI & SI & $\mathrm{R}$ & $\mathrm{S}$ & $\mathrm{S}$ & $\mathrm{R}$ & $\mathrm{R}$ \\
\hline 15 & 301 & S. intermedius & $\mathrm{R}$ & SI & $\mathrm{S}$ & $\mathrm{R}$ & $\mathrm{S}$ & $\mathrm{R}$ & $\mathrm{S}$ & $\mathrm{S}$ & SI & $\mathrm{S}$ \\
\hline 16 & 324 & S. kloosi & $\mathrm{R}$ & $\mathrm{R}$ & SI & $\mathrm{R}$ & $\mathrm{S}$ & $\mathrm{R}$ & $\mathrm{S}$ & $\mathrm{S}$ & $\mathrm{R}$ & $\mathrm{S}$ \\
\hline 17 & 068-1 & S. kloosi & $\mathrm{S}$ & S I & $\mathrm{S}$ & $\mathrm{S}$ & $\mathrm{R}$ & $\mathrm{S}$ & $\mathrm{S}$ & $\mathrm{R}$ & $\mathrm{S}$ & $\mathrm{S}$ \\
\hline 18 & $\mathrm{O} 34$ & E. faecium & SI & $\mathrm{R}$ & $\mathrm{R}$ & SI & $\mathrm{R}$ & $\mathrm{S}$ & $\mathrm{S}$ & SI & $\mathrm{R}$ & $\mathrm{R}$ \\
\hline 19 & OO1 & E. faecium & $\mathrm{R}$ & $\mathrm{S}$ & $\mathrm{S}$ & $\mathrm{S}$ & $\mathrm{R}$ & $\mathrm{S}$ & $\mathrm{S}$ & SI & $\mathrm{R}$ & SI \\
\hline 20 & $508-2$ & E. faecium & $\mathrm{R}$ & $\mathrm{S}$ & $\mathrm{S}$ & $\mathrm{R}$ & $\mathrm{R}$ & $\mathrm{S}$ & $\mathrm{S}$ & $\mathrm{R}$ & $\mathrm{R}$ & S I \\
\hline 21 & $538 s$ & E. faecium & S I & $\mathrm{S}$ & $\mathrm{S}$ & S I & $\mathrm{R}$ & $\mathrm{S}$ & $\mathrm{S}$ & $\mathrm{R}$ & $\mathrm{R}$ & $\mathrm{R}$ \\
\hline 22 & $546 s$ & E. faecium & $\mathrm{R}$ & $\mathrm{S}$ & $\mathrm{S}$ & S I & $\mathrm{S}$ & $\mathrm{S}$ & $\mathrm{S}$ & $\mathrm{S}$ & $\mathrm{S}$ & $\mathrm{S}$ \\
\hline 23 & O81 & E.faecium & $\mathrm{S}$ & $\mathrm{S}$ & $\mathrm{S}$ & $\mathrm{S}$ & $\mathrm{S}$ & $\mathrm{R}$ & $\mathrm{S}$ & $\mathrm{S}$ & $\mathrm{R}$ & $\mathrm{S}$ \\
\hline 24 & $\mathrm{O} 38$ & S. intermedius & $\mathrm{S}$ & $\mathrm{R}$ & SI & $\mathrm{S}$ & $\mathrm{R}$ & $\mathrm{R}$ & $\mathrm{S}$ & $\mathrm{R}$ & $\mathrm{R}$ & SI \\
\hline 25 & O78-s & S. intermedius & $\mathrm{S}$ & $\mathrm{S}$ & $\mathrm{S}$ & $\mathrm{S}$ & $\mathrm{R}$ & $\mathrm{S}$ & $\mathrm{S}$ & SI & $\mathrm{S}$ & $\mathrm{S}$ \\
\hline 26 & O46 & S. epidermidis & $\mathrm{S}$ & $\mathrm{S}$ & $\mathrm{S}$ & $\mathrm{S}$ & $\mathrm{S}$ & $\mathrm{R}$ & $\mathrm{S}$ & $\mathrm{S}$ & $\mathrm{S}$ & $\mathrm{S}$ \\
\hline 27 & 528 & S. kloosi & $\mathrm{S}$ & S I & $\mathrm{S}$ & $\mathrm{S}$ & $\mathrm{S}$ & $\mathrm{R}$ & $\mathrm{S}$ & $\mathrm{S}$ & $\mathrm{S}$ & $\mathrm{S}$ \\
\hline
\end{tabular}

Enr (enrofloxacino); A (ampicilina); Amc (Amoxicilina+Acido clavulánico); Cip (ciprofloxacino); T (Tetraciclina); Sxt (sulfa+trimetropin); Van (Vancomicina); D (doxiciclina); Ox(oxicilina); Sul (Sulperazone). R = resistente; SI: sensibilidad intermedia; $\mathrm{S}=$ sensible. 
dos especies nosocomiales con mayor frecuencia de aislamiento corresponden a: E. faecium y E. cloacae coincidiendo con lo obtenido en un estudio anterior (datos no publicados). Primera conclusión: el Material y Método empleado en este estudio permite aislar e identificar cepas nosocomiales ambientales, por lo cual podría ser considerado para posteriores estudios en estos y otros recintos de atención veterinaria de nuestro país.

En relación al estudio de susceptibilidad antimicrobiana, como se deduce de las Tablas 3 y 4, en las bacterias Gram (+) se puede observar que todas las cepas son resistentes a lo menos a uno de los antimicrobianos probados y que el $85 \%$ (23/27) de las cepas son resistentes a dos o más antimicrobianos, es decir son multiresistentes. Se observa una alta resistencia a quinolonas: el $41 \%(11 / 27)$ y $30 \%(8 / 27)$ de las cepas presentan resistencia a enrofloxacino y a ciprofloxacino, respectivamente. El 56\% (15/27) de las cepas son resistentes a ampicilina, el $63 \%$ (17/27) son resistentes a tetraciclina y presentan un comportamiento similar frente a doxiciclina. Por otra parte, todas las cepas de E.faecium no son vancomicina-resistentes, sugiriéndose que el uso de este antimicrobiano en la terapia aun puede ser eficaz. Finalmente, estos resultados sugieren que las cepas Gram $(+)$ existentes en la sede Bilbao presentan un grado mayor de multiresistencia, respecto de las existentes en la sede Facultad.

En relación a las cepas Gram (-) es posible observar que el 71\% (15/21) de las cepas son multiresistentes. Es de interés señalar que el 11\% $(3 / 21)$ de las cepas aisladas presenta resistencia sólo a un antimicrobiano y que igual porcentaje de las cepas aisladas son sensibles a todos los antimicrobianos probados. Destaca la alta resistencia a ampicilina (71\%), seguido de la resistencia a Sulfa/trimetropin y a Amoxicilina/ Acido Clavulánico (62\%).

Llama la atención, que $3 / 5$ de las cepas de E.coli $(60 \%)$ aisladas sean resistentes al menos a 6 de los 8 antimicrobianos probados y que cuatro de las nueve cepas de E. cloacae (44\%) sean

Tabla 4. Sensibilidad antimicrobiana en aislados Gram (-). Sedes Bilbao y Facultad (oscuro)

\begin{tabular}{|c|c|c|c|c|c|c|c|c|c|c|}
\hline $\mathbf{N}^{\mathbf{o}}$ & Aislado & Especie & Enr & $\mathbf{A}$ & Amc & Cip & $\mathbf{T}$ & Sxt & D & Sul \\
\hline 28 & $335-\mathrm{m}$ & E. cloacae & $\mathrm{S}$ & $\mathrm{R}$ & $\mathrm{R}$ & $\mathrm{S}$ & SI & $\mathrm{S}$ & SI & $\mathrm{S}$ \\
\hline 29 & $002-2$ & E. cloacae & $\mathrm{S}$ & $\mathrm{R}$ & $\mathrm{R}$ & $\mathrm{S}$ & $\mathrm{S}$ & $\mathrm{S}$ & S I & $\mathrm{S}$ \\
\hline 30 & $015-2$ & E. cloacae & $\mathrm{S}$ & $\mathrm{S}$ & $\mathrm{S}$ & $\mathrm{S}$ & $\mathrm{S}$ & $\mathrm{S}$ & $\mathrm{S}$ & $\mathrm{S}$ \\
\hline 31 & $016-3$ & E. cloacae & $\mathrm{S}$ & $\mathrm{S}$ & S I & $\mathrm{S}$ & $\mathrm{S}$ & $\mathrm{S}$ & S I & $\mathrm{S}$ \\
\hline 32 & $017-3$ & E. cloacae & $\mathrm{S}$ & $\mathrm{S}$ & $\mathrm{S}$ & $\mathrm{S}$ & $\mathrm{S}$ & $\mathrm{R}$ & $\mathrm{S}$ & $\mathrm{S}$ \\
\hline 33 & $346-\mathrm{s}$ & E. cloacae & SI & $\mathrm{R}$ & $\mathrm{R}$ & $\mathrm{S}$ & $\mathrm{S}$ & $\mathrm{R}$ & $\mathrm{R}$ & $\mathrm{S}$ \\
\hline 34 & 348-m & E. cloacae & SI & $\mathrm{R}$ & $\mathrm{R}$ & $\mathrm{S}$ & $\mathrm{S}$ & $\mathrm{R}$ & $\mathrm{R}$ & $\mathrm{S}$ \\
\hline 35 & $354-\mathrm{s}$ & E. cloacae & $\mathrm{R}$ & $\mathrm{R}$ & $\mathrm{R}$ & $\mathrm{R}$ & SI & $\mathrm{S}$ & $\mathrm{R}$ & $\mathrm{S}$ \\
\hline 36 & 310 & E. cloacae & $\mathrm{R}$ & $\mathrm{R}$ & $\mathrm{R}$ & $\mathrm{R}$ & $\mathrm{R}$ & $\mathrm{S}$ & $\mathrm{R}$ & $\mathrm{S}$ \\
\hline 37 & $360-\mathrm{m}$ & P. agglomerans & $\mathrm{S}$ & $\mathrm{S}$ & $\mathrm{S}$ & $\mathrm{S}$ & $\mathrm{S}$ & $\mathrm{S}$ & SI & $\mathrm{S}$ \\
\hline 38 & $020-2$ & E. coli & $\mathrm{R}$ & $\mathrm{R}$ & S I & $\mathrm{R}$ & $\mathrm{R}$ & $\mathrm{R}$ & $\mathrm{R}$ & $\mathrm{S}$ \\
\hline 39 & $025-2$ & E. coli & $\mathrm{R}$ & $\mathrm{R}$ & S I & $\mathrm{R}$ & $\mathrm{R}$ & $\mathrm{R}$ & $\mathrm{R}$ & $\mathrm{R}$ \\
\hline 40 & $026-2$ & E. coli & $\mathrm{S}$ & $\mathrm{S}$ & $\mathrm{S}$ & $\mathrm{S}$ & $\mathrm{S}$ & $\mathrm{R}$ & S I & $\mathrm{S}$ \\
\hline 41 & $031-2$ & E. coli & $\mathrm{R}$ & $\mathrm{R}$ & $\mathrm{R}$ & $\mathrm{R}$ & $\mathrm{R}$ & $\mathrm{R}$ & $\mathrm{R}$ & $\mathrm{S}$ \\
\hline 42 & 314 & C. freundii & $\mathrm{S}$ & $\mathrm{R}$ & $\mathrm{R}$ & $\mathrm{S}$ & $\mathrm{S}$ & $\mathrm{R}$ & SI & $\mathrm{S}$ \\
\hline 43 & 077M & P. aeruginosa & $\mathrm{R}$ & $\mathrm{R}$ & $\mathrm{R}$ & $\mathrm{S}$ & $\mathrm{R}$ & $\mathrm{R}$ & $\mathrm{R}$ & $\mathrm{S}$ \\
\hline 44 & 325 & P. aeruginosa & $\mathrm{S}$ & $\mathrm{R}$ & $\mathrm{R}$ & $\mathrm{S}$ & SI & $\mathrm{R}$ & $\mathrm{R}$ & $\mathrm{S}$ \\
\hline 45 & 322 & P. aeruginosa & SI & $\mathrm{R}$ & $\mathrm{R}$ & $\mathrm{S}$ & $\mathrm{R}$ & $\mathrm{R}$ & $\mathrm{R}$ & $\mathrm{S}$ \\
\hline 46 & 323 & P. aeruginosa & $\mathrm{S}$ & $\mathrm{R}$ & $\mathrm{R}$ & $\mathrm{S}$ & $\mathrm{R}$ & $\mathrm{R}$ & $\mathrm{R}$ & $\mathrm{S}$ \\
\hline 47 & $035-2$ & A. baumanni & $\mathrm{S}$ & $\mathrm{R}$ & $\mathrm{R}$ & $\mathrm{S}$ & $\mathrm{S}$ & $\mathrm{S}$ & $\mathrm{S}$ & $\mathrm{S}$ \\
\hline 48 & O14 & E. coli & $\mathrm{S}$ & $\mathrm{S}$ & $\mathrm{S}$ & $\mathrm{S}$ & $\mathrm{S}$ & $\mathrm{R}$ & SI & $\mathrm{S}$ \\
\hline
\end{tabular}

Enr (enrofloxacino); A (ampicilina); Amc (Amoxicilina+Acido clavulánico); Cip (ciprofloxacino); T (Tetraciclina); Sxt (sulfa+trimetropin); D (doxiciclina); Sul (Sulperazone); R = resistente; $\mathrm{SI}=$ sensibilidad intermedia; $\mathrm{S}=$ sensible. 
resistentes al menos a 4 antimicrobianos. Por último, en comparación a las cepas Gram $(+)$, se apreciaría una menor resistencia a quinolonas: Enrofloxacino (29\%) y Ciprofloxacino (24\%).

Lo anterior involucra una preocupación inminente, pues en este estudio preliminar se ha podido constatar: (1) una situación similar a lo observado en medicina humana, donde los principales agentes bacterianos Gram positivos aislados son E. faecium (DeLisle y Perl, 2003) y entre los bacilos Gram negativos no fermentadores Acinetobacter baumannii y Pseudomonas aeruginosa (Héritier y col., 2005; Tsakris y col., 2000), como también Escherichia coli (Bean y col., 2008) como bacilos fermentadores y (2) una alta resistencia a los antimicrobianos utilizados en las posibles terapias, situación que amerita continuar con este tipo de estudios en los recintos utilizados como recintos hospitalarios de pequeños animales.

\section{REFERENCIAS}

1.- ALPUCHE C, DAZA C. 2002. Infecciones nosocomiales por bacterias Gram negativas resistentes a cefalosporinas de espectro extendido: asociación de dos peligrosos enemigos. Enfermedades Infecciosas y Microbiología 22(4): 192-199.

2.- BEAN D, KRAHE D, WAREHAM D. 2008. Antimicrobial resistance in community and nosocomial Escherichia coli urinary tract isolates, London 20052006. Ann Clin Microbiol Antimicrob 7(1): 13.

3.- BOERLIN P, EUGSTER S, GASCHEN F, STRAUB R, SCHAWALDER P. 2001. Transmission of opportunistic pathogens in a veterinary teaching hospital. Veterinary Microbiology 82(4): 347-359.

4.- CUNY C, KUEMMERLE J, STANEK C, WILLEY B, STROMMENGER B, WITTE W. 2006. Emergence of MRSA infections in horses in a veterinary hospital: Strain characterisation and comparison with MRSA from humans. Euro Surveill 11(1): 44-47.

5.- EMORY T, GAYNES R. 1993. An overview of nosocomial infections, including the role of the microbiology laboratory. Clinical Microbiology Reviews 6(4): 428442.

6.- FRANCEY T, GASCHEN F, NICOLET J, BURNENS A. 2000. The role of Acinetobacter baumannii as a nosocomial pathogen for dogs and cats in an intensive care unit. Journal of Veterinary Internal Medicine.
14(2): 177-183.

7.- HERITIER C, DUBOUIX A, POIREL L, MARTY N, NORDMANN P. 2005. A nosocomial outbreak of Acinetobacter baumannii isolates expressing the carbapenem-hydrolysing oxacillinase OXA-58. J Antimicrob Chemotherapy 55(1): 115-118.

8.- HUNT C. 1998. The emergence of enterococci as a cause of nosocomial infection. British Journal of Biomedical Science 55(2): 149-156.

9.- JOHNSON J. 2002. Nosocomial infections. Veterinary Clinics of North America: Small Animal Practice. 32(5): 1101-1126.

10.- LEMMEN S, HAFNER H, ZOLLDANN D, AMEDICK G, LUTTICKEN R. 2001. Comparison of two sampling methods for the detection of gram-positive and gramnegative bacteria in the environment: moistened swabs versus Rodac plates. International Journal of Hygiene and Environmental Health 203(3): 245-248.

11.- LEONARD F, MARKEY B. 2008. Meticillin-resistant Staphylococcus aureus in animals: A review. Vet Journal 175 (1): 27-36. NATIONAL COMMITTE FOR CLINICAL LABORATORY STANDARDS. 1997. Performance standards for antimicrobial dik susceptibility test. Appproved standards. NCCLS document M2-A3. National Committe for Clinical Laboratory Standards.

12.- PRESCOTT J, HANNA W, REID-SMITH R, DROST K. 2002. Antimicrobial drug use and resistance in dogs. The Canadian Veterinary Journal 43(2): 107-116.

13.- ORGANIZACION MUNDIAL DE LA SALUD. 2003. Epidemiología de las infecciones nosocomiales. Prevención de las infecciones nosocomiales. pp 4-8. Segunda Edición. Malta. OMS.

14.- SÁNCHEZ M, BELLO H, DOMÍNGUEZ M, MELLA S, ZEMELMAN R, GONZÁLEZ G. 2006. Transferencia de de $\beta$-lactamasas de espectro extendido desde cepas hospitalarias de Klebsiella pneumoniae a otras especies de enterobacterias. Rev Med Chile 134(4): 415-420.

15.- SEGUIN J, WALKER R, CARON J, KLOOS W, GEORGE C, HOLLIS R, JONES R, PFALLER M. 1999. Methicillin-resistant Staphylococcus aureus outbreak in a veterinary teaching hospital: Potencial human-to-animal transmission. Journal of Clinical Microbiology 37(5): 1459-1463.

16.- TOMLIN J, PEAD J, LLOYD D, HOWELL S, HARTMANN F, JACKSON H, MUIR P. 1999. Methicillin-resistant Staphylococcus aureus infections in 11 dogs. Veterinary Record 144(3): 60-64.

17.- TSAKRIS A, POURNARAS S, WOODFORD $\mathrm{N}$, PALEPOU M, BABINI G, DOUBOYAS J, LIVERMORE D. 2000. Outbreak of infections caused by Pseudomonas aeruginosa producing VIM1 Carbapenemase in Greece. Journal of Clinical Microbiology 38 (3): 1290-1292. 